\section{Propriedades psicométricas do Internet Addiction Test em estudantes de Montes Claros, Minas Gerais, Brasil}

\author{
Psychometric properties of the Internet Addiction \\ Test in students in Montes Claros, Minas Gerais \\ State, Brazil
}

\section{Propiedades psicométricas del Internet Addiction Test en estudiantes de Montes Claros, Minas Gerais, Brasil}

Alexandre Botelho Brito 1

Lucinéia de Pinho 2

Maria Fernanda Santos Figueiredo Brito 2

Romerson Brito Messias 3

Kewla Dias Pires Brito 4

Carolina Amaral Oliveira Rodrigues 5

Vivianne Margareth Chaves Pereira Reis 6

Marise Fagundes Silveira ${ }^{7}$

doi: 10.1590/0102-311X00212619

\section{Resumo}

O objetivo foi avaliar as propriedades psicométricas da versão traduzida para o português do Internet Addiction Test (IAT) em estudantes. Estudo metodológico, cuja população foi constituída por estudantes dos ensinos Médio e Superior de Montes Claros, Minas Gerais, Brasil. Foram avaliadas sensibilidade psicométrica, validade de construto (fatorial, convergente, discriminante e teste de hipótese), validade de critério (concorrente) e confiabilidade (consistência interna e reprodutibilidade). Participaram 2.519 estudantes, sendo 29,6\% do Ensino Médio. Foi ajustado um modelo de medida trifatorial - "preocupação emocional e cognitiva com a Internet", "problemas de gerenciamento de tempo" e "problemas de desempenho" -, o qual obteve indices satisfatórios de adequação e estrutura estável nas subamostras independentes. $A$ validade convergente foi próxima ao recomendado (variância extraída média $=0,32,0,41$ e 0,45 e valores de confiabilidade composta iguais 0,84, 0,81 e 0,71); as validades discriminante e concorrente foram adequadas. Foram igualmente adequadas a consistência interna $($ alfa $=0,906)$ e a reprodutibilidade (kappa $=0,73$ e coeficiente de correlação intraclasse $=0,90)$. A versão traduzida para o português do IAT apresentou niveis satisfatórios de validade, confiabilidade e estabilidade em amostras independentes de estudantes.

Estudo de Validação; Internet; Estudantes

\author{
Correspondência \\ A. B. Brito \\ Rua Dois 300, Montes Claros, MG 39404-058, Brasil. \\ alexandre.brito@ifnmg.edu.br \\ 1 Instituto Federal do Norte de Minas Gerais, Montes Claros, \\ Brasil. \\ 2 Programa de Pós-graduação em Cuidados Primários em \\ Saúde, Universidade Estadual de Montes Claros, Montes Claros, \\ Brasil. \\ 3 Departamento de Saúde Mental e Coletiva, Universidade \\ Estadual de Montes Claros, Montes Claros, Brasil. \\ 4 Instituto Federal do Norte de Minas Gerais, Pirapora, Brasil. \\ 5 Universidade Estadual de Montes Claros, Montes Claros, \\ Brasil. \\ 6 Departamento de Educação Física, Universidade Estadual de \\ Montes Claros, Montes Claros, Brasil. \\ 7 Programa de Pós-graduação em Ciências da Saúde, \\ Universidade Estadual de Montes Claros, Montes Claros, Brasil.
}




\section{Introdução}

Com o avanço tecnológico ocorrido nas últimas décadas, a Internet tornou-se uma das formas mais importantes no mundo atual de acesso a informações e conhecimentos científicos, comunicação, entretenimento e trabalho 1 . O uso dessa rede vem crescendo exponencialmente e tornou-se popular nos hábitos de vida dos seus usuários 2. Paralelamente à popularidade e aos seus benefícios, emergiram efeitos prejudiciais do seu uso de forma desadaptativa, como a adição em Internet 3 .

A adição em Internet tem sido considerada uma epidemia do século XXI 3 e representa um problema mundial de saúde pública 4 . Esse distúrbio está associado a comprometimentos mentais, como depressão, ansiedade e problemas obsessivo-compulsivos 5,6,7 e dependência de substâncias, além de comprometimento físico ${ }^{8}$. Na vida escolar, a adição em Internet pode contribuir para menor produtividade do estudante ${ }^{8}$, absenteísmo e evasão ${ }^{9}$, com impacto social significativo.

A adição em Internet constitui um padrão de comportamento mal adaptativo de uso da Internet e caracteriza-se como um transtorno de controle de impulso ou dependência, cujas cognições associadas resultam em consequências negativas na vida pessoal e profissional dos usuários 4,10 . $\mathrm{O}$ padrão de uso excessivo de Internet pode causar dependência de jogos online, redes sociais, pornografia online e de smartphone 8 , o que resulta na literatura em uma diversidade de termos considerados para a sua avaliação 11.

Nessa perspectiva, instrumentos de rastreamento da adição em Internet válidos e confiáveis são necessários para a sua identificação e caracterização. Entre os instrumentos utilizados, destaca-se o Internet Addiction Test (IAT) 12,13,14, que foi desenvolvido por Kimberly Young em 1998 15, traduzido para o português no Brasil, em 2012 16, e traduzido e validado para o português em Portugal, em 201417. O IAT também foi validado em vários países, como Espanha 18, Coreia do Sul 19, Malásia 20, China 14 e Estados Unidos 12, que indicaram propriedades psicométricas satisfatórias quando empregado em diferentes populações. No que se refere à confiabilidade, foi demonstrado que a escala IAT reflete consistentemente o construto que está medindo e gera resultados semelhantes quando aplicado em situações diferentes, conferindo a sua estabilidade temporal. Foi observada consistência interna adequada, com valores do coeficiente de alfa de Cronbach entre 0,89 13 e 0,93 21. Quanto à reprodutibilidade, os estudos observaram indicadores superiores a 0,60 18 .

Com relação à estrutura fatorial, a versão original foi proposta com um modelo unifatorial ${ }^{15}$, como encontrado no estudo de Pontes et al. 17. Entretanto, na literatura, há variações, sendo observadas estruturas com dois 18 , três 13 , cinco 22 e seis 20 fatores, que refletem construtos relacionados a "isolamento e problemas sociais", "gerenciamento de tempo e desempenho", "negligência no trabalho" e "falta de controle e substituição da realidade".

Estudos identificaram validade de critério concorrente do IAT ao correlacionarem os seus escores com aqueles dos instrumentos Internet-Related Problem Scale (IRPS) 23, Compulsive Internet Use Scale (CIUS) 22, Loneliness Scale (UCLA) 13 e Inventário de Depressão de Beck - Segunda Edição (BDI-II) 17, bem como com o tempo de uso diário da Internet 21. Apesar da evidência de associação entre os escores mais altos do IAT e outras psicopatologias, como a depressão, alguns autores ponderam cautela na interpretação, uma vez que não foram relacionados à gravidade ou à duração dos sintomas daqueles clinicamente diagnosticados com dependência da Internet 24 .

Embora o IAT tenha apresentado níveis adequados de validade e confiabilidade em estudantes de outros países, não terá, necessariamente, o mesmo desempenho em grupos populacionais inseridos em diferentes contextos daqueles em que foi aplicado anteriormente 25, pois o uso da Internet pode ser influenciado por fatores demográficos, sociais, culturais e econômicos 5. Ademais, embora no Brasil já tenham sido realizadas tradução, análise de equivalência semântica e de consistência interna deste instrumento, sua sensibilidade, validade de construto e de critério, assim como sua reprodutibilidade ainda não foram avaliadas 16 . Portanto, no contexto nacional, há uma lacuna quanto às propriedades psicométricas do IAT, o que inscreve o presente estudo como importante contribuição à compreensão desse fenômeno.

Nesse contexto, o objetivo do presente estudo foi avaliar a validade e confiabilidade da versão traduzida para o português do IAT em uma amostra de estudantes brasileiros. 


\section{Métodos}

\section{Tipo de estudo e população-alvo}

O presente estudo utiliza dados da Pesquisa Estudantes Online: Uso e Dependência da Internet, cujo objetivo principal foi estimar a prevalência da adição em Internet. A população desse inquérito foi constituída por estudantes em instituições públicas e privadas regularmente matriculados no Ensino Médio $\left(\mathrm{N}_{\text {público }}=12.087\right.$ e $\left.\mathrm{N}_{\text {privado }}=4,129\right)$ e Superior $\left(\mathrm{N}_{\text {público }}=6.666\right.$ e $\left.\mathrm{N}_{\text {privado }}=19.217\right)$ da zona urbana do Município de Montes Claros, norte de Minas Gerais, Brasil.

\section{Tamanho da amostra e seleção das unidades amostrais}

Para o estudo transversal, o tamanho da amostra foi determinado adotando-se os seguintes parâmetros: prevalência de adição em Internet de 13\% 26, nível de 95\% de confiança e margem de erro de $2 \%$, com correção para população finita. Estabeleceu-se também um acréscimo de 15\% para compensar possíveis perdas, e foi realizada correção para o efeito de delineamento $($ deff $=2,0)$ por se tratar de um plano amostral por conglomerado. $\mathrm{O}$ tamanho da amostra estimado foi de 2.500 estudantes, sendo 963 do Ensino Médio e 1.537 do Ensino Superior, estratificados proporcionalmente entre as instituições públicas e privadas.

Para o estudo metodológico de análise das propriedades psicométricas do IAT, o tamanho da amostra foi estabelecido considerando os parâmetros para a validade de construto e de reprodutibilidade. Para a validade de construto, adotou-se a estimativa de dez respondentes por item do instrumento $(20 \times 10=200$ participantes), bem como dez respondentes para cada parâmetro livre a ser estimado no modelo ( $66 \times 10=660$ participantes) 27 . Para avaliar a reprodutibilidade, estabeleceu-se a participação de, no mínimo, $10 \%$ da amostra do estudo transversal (250 participantes) 28.

A seleção da amostra foi do tipo probabilístico por conglomerado em um único estágio no Ensino Médio e em três estágios no Ensino Superior. No primeiro estágio, por probabilidade proporcional ao tamanho (PPT), foram sorteadas as instituições de ensino (cinco do Ensino Médio e quatro do Ensino Superior). No Ensino Médio, foram selecionadas todas as turmas das instituições sorteadas. No Ensino Superior, no segundo estágio, foram sorteados, por PPT, os cursos estratificados pela área de conhecimento (saúde/biológicas, exatas, humanas e sociais aplicadas); no terceiro estágio, por amostragem aleatória simples (AAS), foi realizado o sorteio de duas turmas por curso. Em ambos os níveis de ensino, todos os alunos das turmas selecionadas foram convidados a participar do estudo.

Para verificar a reprodutibilidade do IAT, foram selecionadas, por AAS, duas instituições, uma do Ensino Médio e outra do Ensino Superior. Em seguida, também por AAS, sortearam-se quatro turmas no Ensino Médio e cinco no Ensino Superior. Todos os alunos dessas turmas foram convidados a participar da pesquisa. O questionário foi aplicado e reaplicado em um intervalo de duas semanas.

Utilizou-se como critério de inclusão ser estudante regularmente matriculado na turma selecionada. Como critério de exclusão, considerou-se o fato de o estudante não estar presente no dia agendado para a coleta de dados.

\section{Coleta de dados}

A coleta de dados foi realizada entre o segundo semestre de 2016 e o primeiro semestre de $2017 \mathrm{em}$ sala de aula, por uma equipe multiprofissional das áreas de medicina, psicologia, enfermagem, nutrição e exatas e por estudantes de iniciação científica previamente capacitados.

Para caracterização do perfil dos participantes, utilizou-se um questionário que contemplou as características demográficas (sexo, idade, estado civil), socioeconômicas (nível de ensino, se exerce atividade profissional e classe econômica) e perfil de uso de Internet (turno de utilização, frequência e propriedade de aparelho com acesso à Internet).

Foi utilizada a versão traduzida para o português do instrumento IAT 16, que é constituído por 20 itens, com opções de respostas em escala do tipo Likert, cuja pontuação total é obtida pela soma das respostas dos seus itens, podendo variar de 20 a 100 pontos. Por meio dessa pontuação, é possível classificar o indivíduo em: usuário mediano, que tem controle completo sobre seu uso (20 a 39 pontos); 
usuário problemático, que tem problemas ocasionais (40 a 69 pontos) e usuário com adição, que tem problemas significativos por causa do uso da Internet (70 a 100 pontos) 15.

\section{Análise estatística}

Inicialmente foi realizada análise descritiva das variáveis de caracterização do perfil demográfico, socioeconômico e de utilização da Internet por meio das medidas de frequência absoluta e relativa, com correção pelo efeito de desenho. As evidências de validade do IAT foram avaliadas pela análise da sensibilidade psicométrica, validade de construto (fatorial, convergente, discriminante e teste de hipótese), validade de critério concorrente e confiabilidade (análise da consistência interna e reprodutibilidade).

\section{- Sensibilidade psicométrica}

Utilizaram-se as medidas de tendência central, dispersão, assimetria e curtose dos itens do IAT. Os itens com coeficientes de assimetria $(\mathrm{sk}<3)$ e curtose $(\mathrm{ku}<7)$ foram considerados com adequada sensibilidade 27 .

\section{- Validade de construto (fatorial, convergente, discriminante e teste de hipótese)}

Para avaliar a estrutura fatorial do IAT, foi realizada a análise fatorial exploratória (AFE), seguida pela análise fatorial confirmatória (AFC). Na AFE, adotou-se a extração dos fatores pelo método das componentes principais com rotação ortogonal Varimax (máxima variância de fatores). Os fatores comuns retidos foram aqueles que apresentaram autovalor superior a 1, em consonância com o diagrama de declividade e com a porcentagem de variância explicada concomitantemente com a análise da coerência dos itens. Foi avaliado o comportamento das cargas dos itens nos fatores, adotando-se como critério de exclusão cargas abaixo de 0,40. Para itens que apresentaram carga fatorial a partir de 0,40 em mais de um fator, o critério de alocação foi por meio da análise de coerência do item. Para avaliar a adequação dos dados, utilizou-se a medida de Kaiser-Meyer-Olkin (KMO), para a qual adotou-se valor superior a 0,5. A presença de correlação entre as variáveis foi medida pela análise da matriz de correlações e o teste de esfericidade de Bartlett, considerando-se nível de significância de 0,05 29.

A AFC foi utilizada para ratificar a estrutura dimensional extraída na AFE. Para avaliar a qualidade do modelo de medida ajustado, utilizaram-se a razão entre o qui-quadrado e os graus de liberdade ( $\chi^{2 / g .1 .), ~ o s ~ i ́ n d i c e s ~ d e ~ q u a l i d a d e ~ d e ~ a j u s t e ~(G F I), ~ d e ~ T u c k e r-L e w i s ~(T L I), ~ d e ~ a j u s t e ~ c o m p a r a t i v o ~ d e ~}$ Bentler (CFI) e a raiz do erro quadrático médio de aproximação (RMSEA). Os parâmetros considerados para avaliar o ajuste global do modelo foram $\chi^{2} /$ g.l. $<$ 5; CFI, GFI e TLI $\geq 0,9$; e RMSEA < 0,10 30.

Após o ajuste do melhor modelo, foi conduzida a análise multigrupos para verificar a sua estabilidade (invariância do modelo). Para tanto, a amostra global foi dividida em duas subamostras aleatórias de 1.259 e 1.260 estudantes 31 . Ajustou-se o modelo individualmente em cada uma das subamostras, e a sua invariância foi avaliada por comparação do modelo livre (com cargas fatoriais e variâncias/ covariâncias dos fatores livres) com cada um dos três modelos a seguir: um modelo no qual foram fixadas as cargas fatoriais, outro no qual foram fixadas as cargas fatoriais e as variâncias/covariâncias dos dois grupos e o último modelo, no qual foram fixados as cargas fatoriais, as variâncias/covariâncias e os resíduos dos dois grupos. A significância estatística da diferença entre os modelos comparados foi avaliada por meio do teste qui-quadrado $\left(\chi^{2}\right)$, como descrito por Marôco ${ }^{31}$.

A validade de construto convergente foi estimada pela variância extraída média (VEM) e pela confiabilidade composta (CC). Valores de VEM $\geq 0,5$ e de CC $\geq 0,7$ foram considerados adequados 31 . A validade convergente foi também avaliada por meio da análise das cargas fatoriais dos itens do IAT. Valores de cargas fatoriais $\geq 0,50$ foram indicadores de validade convergente 32 .

A validade discriminante foi demonstrada comparando-se as VEMs de dois fatores com o quadrado do coeficiente de correlação entre esses fatores. A evidência de validade discriminante foi confirmada quando as VEMs dos fatores foram superiores ou iguais ao quadrado da correlação entre eles 31 .

O teste de hipótese foi investigado por meio da comparação dos escores do IAT entre subgrupos que hipoteticamente deveriam apresentar níveis distintos de adição em Internet, utilizando-se os 
testes Mann-Whitney e Kruskal-Wallis (nível de significância = 0,05). Esses subgrupos foram definidos a partir de características demográficas (sexo e idade), socioeconômicas (classe econômica) e de perfil de uso da Internet (turno de utilização, frequência de acesso às redes sociais, aos jogos online e para ver/ouvir filmes, músicas e vídeos).

A escala IAT foi considerada com validade de construto quando pelo menos três desses critérios (fatorial, convergente, discriminante e teste de hipótese) apresentaram resultados dentro dos limites considerados satisfatórios.

\section{- Validade de critério concorrente}

A validade de critério concorrente foi avaliada pela correlação entre o escore total do IAT e a autopercepção da adição em Internet, sendo esta avaliada por meio da seguinte pergunta: "Você se considera viciado (adito) em Internet?", com resposta distribuída em uma escala graduada, tipo Likert. Também foi analisada a correlação entre o escore total do IAT e o tempo de utilização da Internet, em horas, nos dias úteis e em fins de semana. Em todas as análises de correlação, adotou-se o coeficiente de correlação de Spearman, visto que as variáveis não apresentaram distribuição normal (teste de Kolmogorov-Smirnov).

\section{- Confiabilidade (análise da consistência interna e reprodutibilidade)}

A confiabilidade do IAT foi avaliada por meio da sua consistência interna e reprodutibilidade. A consistência interna foi testada pelo coeficiente alfa de Cronbach para a escala geral (todos os itens) e para cada um dos fatores extraídos. Foi adotado o valor mínimo de 0,70 para uma consistência interna satisfatória 32 .

Para a análise de reprodutibilidade, foi calculado o escore total do IAT, que posteriormente foi categorizado (usuário mediano, problemático ou adito) nos dois momentos da aplicação. Para estimar a concordância entre as respostas, utilizou-se o coeficiente kappa ponderado, para o IAT categorizado, e o coeficiente de correlação intraclasse (CCI), para o escore total e para os fatores extraídos. A interpretação do coeficiente kappa foi realizada a partir dos critérios propostos por Landis e Koch, seguindo esta escala: sem concordância $(\mathrm{k}<0)$, concordância pobre $(0 \leq \mathrm{k} \leq 0,19)$, concordância razoável $(0,20$ a 0,39$)$, concordância moderada $(0,40 \leq \mathrm{k} \leq 0,59)$, concordância substancial $(0,60 \leq \mathrm{k} \leq 0,79)$ e excelente concordância $(0,80 \leq \mathrm{k} \leq 1,00)$. Para interpretação do CCI, foi adotada a seguinte escala: concordância pobre $(\mathrm{CCI}<0,40)$, concordância satisfatória $(0,40 \leq \mathrm{CCI}<0,75)$ e concordância excelente $(\mathrm{CCI} \geq 0,75) 33$.

\section{- Processamento dos dados}

Os dados foram digitados em duplicata e processados no software SPSS, versão 22.0 (https://www. ibm.com/). A análise fatorial confirmatória foi realizada via software SPSS Amos (https://www.ibm. com/), utilizando o método da máxima verossimilhança 31. Os valores omissos (missing) foram estimados por meio das médias, segundo nível de ensino, idade e sexo dos estudantes, visto que a frequência dos itens do IAT com valores omissos foi pequena $($ mínimo $=2$ e máximo $=7$ ) em face do tamanho $\operatorname{amostral}(\mathrm{n}=2.519)$.

\section{Questões éticas}

Este estudo foi conduzido dentro dos padrões da Resolução no 466/2012, e o projeto desta pesquisa foi aprovado pelo Comitê de Ética em Pesquisa da Universidade Estadual de Montes Claros (protocolo no 1.520.173/2016). Todos os participantes assinaram o Termo de Consentimento Livre e Esclarecido (TCLE). Para os estudantes com idade inferior a 18 anos, foi recolhido o TCLE assinado pelos pais dos entrevistados, juntamente com o Termo de Assentimento Livre e Esclarecido (TALE) igualmente assinado por estes. 


\section{Resultados}

\section{Caracterização da amostra}

Participaram deste estudo 2.519 estudantes, sendo 966 (29,6\%) do Ensino Médio e 1.553 (70,4\%) do Ensino Superior (taxa de resposta obtida igual a 98\%). As médias da idade dos participantes foram, respectivamente, iguais a 16,6 anos $(\mathrm{DP}=1,3)$ e 23,5 anos $(\mathrm{DP}=5,9)$. A maioria era do sexo feminino (60,5\%), solteira (84,6\%), não trabalhava (61,6\%), tinha menos de 25 anos $(73,5 \%)$, pertencia às classes econômicas B e C (42,1\% e 42\% respectivamente), acessava a Internet mais de seis vezes ao dia $(67,7 \%)$, todos os dias da semana $(86,1 \%)$ e no período noturno $(85,7 \%)$. Apenas $0,7 \%$ dos estudantes não era proprietário de algum aparelho com acesso à Internet.

\section{Sensibilidade psicométrica}

A pontuação média nos itens do instrumento IAT variou de 1,6 (Item 19) a 3,4 (Item 1). Os itens do IAT apresentaram valores de assimetria e curtose que indicam uma aproximação de distribuição normal, respectivamente com valores máximos de 1,8 e 2,6, ambos no item 19 (Tabela 1).

\section{Validade de construto (fatorial, convergente, discriminante e teste de hipótese)}

A análise da matriz de correlações demonstrou coeficientes de correlações significativas entre os itens do IAT ( $\mathrm{p}<0,001$ ), cujos valores variaram de 0,16 a 0,63 . Não houve itens excessivamente correlacionados, indicando ausência de multicolinearidade. A análise das comunalidades mostrou valores entre 0,28 e 0,73. Verificou-se fatoriabilidade da matriz dos dados com valor de KMO igual a

\section{Tabela 1}

Medidas descritivas dos itens e do escore total do Internet Addiction Test. Montes Claros, Minas Gerais, Brasil, $2016-2017$.

\begin{tabular}{|c|c|c|c|c|c|}
\hline Item & Média & Mediana & Desvio padrão & Assimetria & Curtose \\
\hline 1 & 3,4 & 3,0 & 1,3 & $-0,3$ & $-1,1$ \\
\hline 2 & 2,3 & 2,0 & 1,2 & 0,7 & $-0,5$ \\
\hline 3 & 1,7 & 1,0 & 1,1 & 1,6 & 1,7 \\
\hline 4 & 2,4 & 2,0 & 1,3 & 0,7 & $-0,7$ \\
\hline 5 & 2,3 & 2,0 & 1,4 & 0,8 & $-0,7$ \\
\hline 6 & 1,6 & 1,0 & 1,0 & 1,7 & 2,2 \\
\hline 7 & 3,0 & 3,0 & 1,4 & 0,1 & $-1,2$ \\
\hline 8 & 1,7 & 1,0 & 1,1 & 1,6 & 1,7 \\
\hline 9 & 1,8 & 1,0 & 1,2 & 1,5 & 1,0 \\
\hline 10 & 2,0 & 1,0 & 1,3 & 1,1 & 0,0 \\
\hline 11 & 2,2 & 2,0 & 1,3 & 0,8 & $-0,5$ \\
\hline 12 & 2,5 & 2,0 & 1,5 & 0,6 & $-1,1$ \\
\hline 13 & 1,7 & 1,0 & 1,1 & 1,7 & 2,1 \\
\hline 14 & 2,4 & 2,0 & 1,4 & 0,7 & $-0,8$ \\
\hline 15 & 2,2 & 2,0 & 1,3 & 0,9 & $-0,3$ \\
\hline 16 & 2,7 & 2,0 & 1,4 & 0,3 & $-1,2$ \\
\hline 17 & 2,4 & 2,0 & 1,4 & 0,6 & $-0,9$ \\
\hline 18 & 1,8 & 1,0 & 1,2 & 1,6 & 1,2 \\
\hline 19 & 1,6 & 1,0 & 1,0 & 1,8 & 2,6 \\
\hline 20 & 1,7 & 1,0 & 1,2 & 1,6 & 1,5 \\
\hline Escore total & 43,3 & 41,0 & 15,1 & 0,7 & 0,1 \\
\hline
\end{tabular}


0,944 e o teste de esfericidade de Bartlett significativo $(\mathrm{p}<0,001)$. A AFE identificou três fatores que explicaram 48,45\% de variância. Na Tabela 2 são apresentados os valores das cargas fatoriais de cada item, por fator. A variância explicada pelo fator $1\left(\mathrm{~F}_{1}\right)$, fator $2\left(\mathrm{~F}_{2}\right)$ e fator $3\left(\mathrm{~F}_{3}\right)$ foi respectivamente $19,72 \%, 18,84 \%$ e $9,89 \%$.

Procedeu-se à inspeção das cargas fatoriais de acordo com os critérios de exclusão estabelecidos, e permaneceram todos os itens do instrumento. Na análise do padrão de cargas cruzadas, observouse que os itens 2, 11, 12 e 15 apresentaram cargas fatoriais adequadas para dois fatores. Nenhum dos itens foi eliminado por essa situação, por ter sido considerado imprescindível ao conteúdo do instrumento. A alocação desses itens foi realizada conforme coerência do item ao fator, sendo o item 2 alocado no $\mathrm{F}_{3}$, e os itens 11,12 e 15 alocados no $\mathrm{F}_{1}$.

$\mathrm{O} \mathrm{F}_{1}$, composto por 11 itens $(3,4,9,10,11,12,13,15,18,19$ e 20), foi intitulado "preocupação emocional e cognitiva com a Internet" e se refere aos sentimentos gerados com o uso da Internet e à preferência do usuário pelo convívio virtual sobre as relações presenciais com familiares e amigos. $\mathrm{O}$

Tabela 2

Cargas fatoriais dos itens do Internet Addiction Test obtidas pela análise fatorial exploratória. Montes Claros, Minas Gerais, Brasil, $2016-2017$.

\begin{tabular}{|c|c|c|c|c|}
\hline Item & Conteúdo & $\mathbf{F}_{1}$ & $\mathbf{F}_{2}$ & $\mathbf{F}_{3}$ \\
\hline 1 & Com que frequência você acha que passa mais tempo na Internet do que pretendia? & 0,026 & 0,670 & 0,159 \\
\hline 2 & Com que frequência você abandona as tarefas diárias para passar mais tempo na Internet? & 0,143 & 0,570 & 0,421 \\
\hline 3 & $\begin{array}{l}\text { Com que frequência você prefere a emoção da Internet ao convívio com seu/sua } \\
\text { namorado(a)/esposo(a)/amigo(a)/pai ou mãe...? }\end{array}$ & 0,478 & 0,117 & 0,277 \\
\hline 4 & Com que frequência você cria relacionamentos com novo(a)s amigo(a)s da Internet? & 0,488 & 0,200 & 0,074 \\
\hline 5 & $\begin{array}{l}\text { Com que frequência outras pessoas em sua vida se queixam sobre a quantidade de tempo } \\
\text { que você passa na Internet? }\end{array}$ & 0,377 & 0,472 & 0,196 \\
\hline 6 & $\begin{array}{l}\text { Com que frequência suas notas ou tarefas da escola pioram por causa da quantidade de } \\
\text { tempo que você fica na Internet? }\end{array}$ & 0,204 & 0,241 & 0,782 \\
\hline 7 & $\begin{array}{l}\text { Com que frequência você acessa a Internet antes de qualquer outra coisa que precise } \\
\text { fazer? }\end{array}$ & 0,168 & 0,671 & 0,197 \\
\hline 8 & $\begin{array}{l}\text { Com que frequência piora o seu desempenho ou produtividade no trabalho/escola por } \\
\text { causa da Internet? }\end{array}$ & 0,192 & 0,236 & 0,796 \\
\hline 9 & $\begin{array}{l}\text { Com que frequência você fica na defensiva ou guarda segredo quando alguém lhe } \\
\text { pergunta o que você faz na Internet? }\end{array}$ & 0,538 & 0,039 & 0,328 \\
\hline 10 & $\begin{array}{l}\text { Com que frequência você bloqueia pensamentos perturbadores sobre sua vida pensando } \\
\text { em se conectar para acalmar-se? }\end{array}$ & 0,611 & 0,184 & 0,170 \\
\hline 11 & Com que frequência você se pega pensando em quando vai entrar na Internet novamente? & 0,580 & 0,468 & 0,059 \\
\hline 12 & Com que frequência você teme que a vida sem a Internet seja chata, vazia e sem graça? & 0,504 & 0,499 & $-0,097$ \\
\hline 13 & $\begin{array}{l}\text { Com que frequência você explode, grita ou se irrita se alguém o(a) incomoda enquanto está } \\
\text { na Internet? }\end{array}$ & 0,593 & 0,230 & 0,078 \\
\hline 14 & Com que frequência você dorme pouco por usar a Internet até tarde da noite? & 0,313 & 0,584 & 0,135 \\
\hline 15 & $\begin{array}{l}\text { Com que frequência você se sente preocupado(a) com a Internet quando está } \\
\text { desconectado(a), imaginando que poderia estar conectado(a)? }\end{array}$ & 0,537 & 0,519 & $-0,011$ \\
\hline 16 & $\begin{array}{l}\text { Com que frequência você se pega dizendo "só mais alguns minutos" quando está } \\
\text { conectado(a)? }\end{array}$ & 0,352 & 0,663 & 0,090 \\
\hline 17 & Com que frequência você tenta diminuir o tempo que fica na Internet e não consegue? & 0,233 & 0,613 & 0,262 \\
\hline 18 & Com que frequência você tenta esconder a quantidade de tempo que está na Internet? & 0,469 & 0,307 & 0,296 \\
\hline 19 & $\begin{array}{l}\text { Com que frequência você opta por passar mais tempo na Internet em vez de sair com } \\
\text { outras pessoas? }\end{array}$ & 0,596 & 0,074 & 0,177 \\
\hline 20 & $\begin{array}{l}\text { Com que frequência você se sente deprimido, mal-humorado, ou nervoso quando } \\
\text { desconectado e esse sentimento vai embora assim que volta a se conectar à Internet? }\end{array}$ & 0,679 & 0,270 & 0,059 \\
\hline \multicolumn{2}{|c|}{ Porcentagem da variância explicada } & 19,72 & 18,84 & 9,89 \\
\hline
\end{tabular}


$\mathrm{F}_{2}$ foi nomeado "problemas de gerenciamento de tempo" sendo composto por seis itens $(1,5,7,14$, $16,17)$ que abordaram a necessidade do controle do tempo de uso da Internet e a sua prioridade ante outras atividades. $\mathrm{OF}_{3}$, denominado "problemas de desempenho", contemplou três itens (2, 6 e 8), que manifestaram as consequências do uso da Internet no desempenho das atividades diárias de casa, na escola e/ou nas atividades profissionais.

A Figura 1 apresenta o modelo trifatorial ajustado pela AFC, as cargas fatoriais dos itens, suas variâncias e as correlações entre os três fatores. Esse modelo apresentou adequada qualidade do ajuste

\section{Figura 1}

Modelo trifatorial do Internet Addiction Test (IAT) ajustado pela análise fatorial confirmatória ( $\chi^{2} / \mathrm{g} . \mathrm{I}$. = 4,866; CFI = 0,964; $\mathrm{GFI}=0,970 ; \mathrm{TLI}=0,956$ e RMSEA $=0,039$ ).

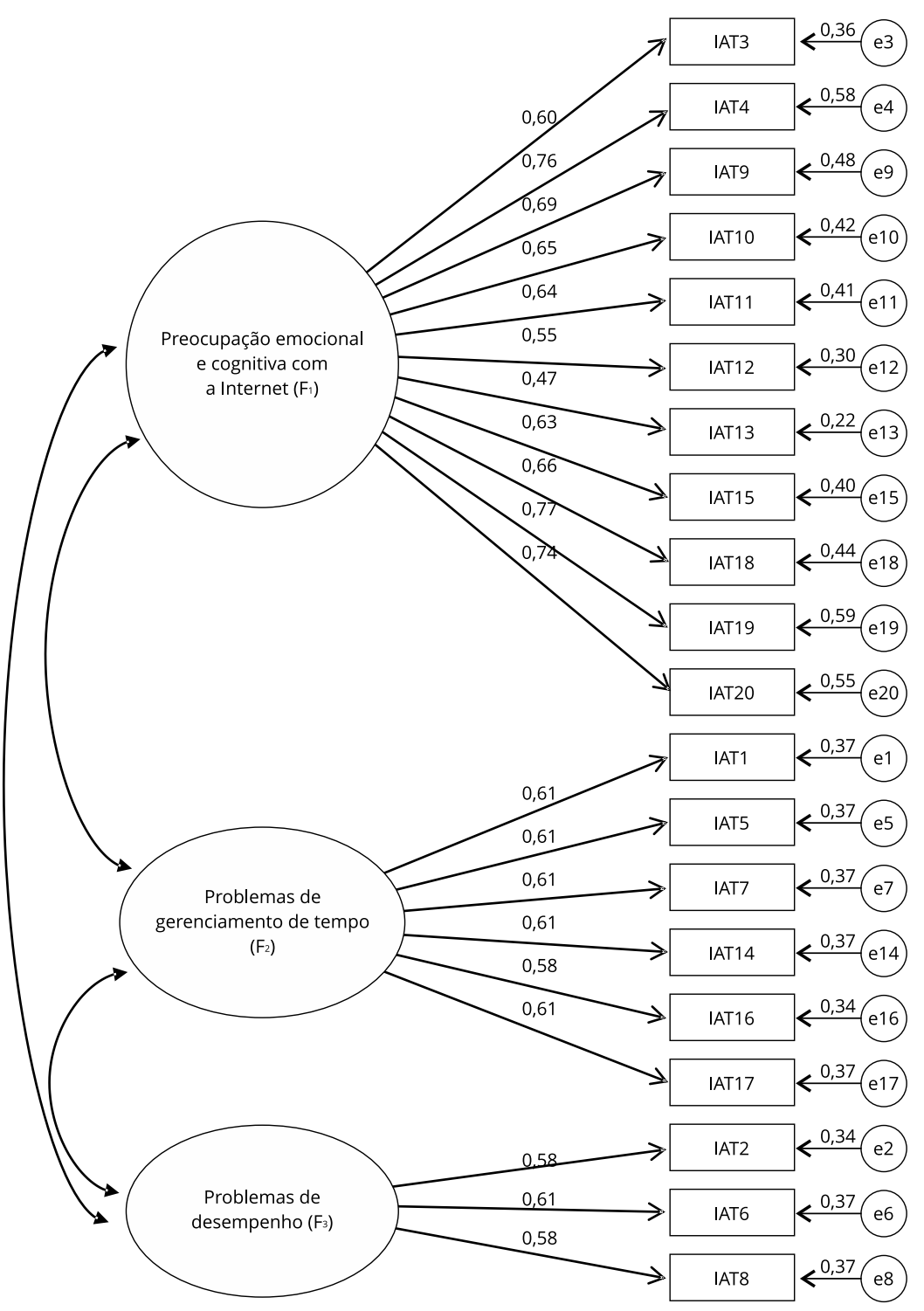

X²/g.I.: qui-quadrado e graus de liberdade; CFI: índice de ajuste comparativo; GFI: índice de qualidade de ajuste; RMSEA: raiz do erro quadrático médio de aproximação; TLI: índice de Tucker-Lewis. 
local, com cargas fatoriais dos itens $(\lambda)$ maiores ou iguais a 0,40 . O ajuste global do modelo foi adequado (Figura 1). Foram constatadas também importantes correlações entre os três fatores do modelo.

A análise de invariância do modelo fatorial encontrado, considerando a mesma estrutura nas duas subamostras de análise, apontou para a inexistência de diferenças significativas entre as cargas fatoriais $\left(\chi^{2}(17)=10,032 ; p=0,902\right)$, entre as covariâncias $\left(\chi^{2}(23)=15,485 ; \mathrm{p}=0,876\right)$ e entre os resíduos $\left(\chi^{2}(54)=59,403 ; \mathrm{p}=0,285\right)$.

Quanto à validade convergente, os valores de VEM foram menores que 0,50, porém os valores de CC foram maiores que 0,7 (Tabela 3). Com relação às cargas fatoriais, apenas o item 13 apresentou carga fatorial inferior a 0,50 (Figura 1).

Observou-se validade discriminante dos fatores $\mathrm{F}_{1}$ e $\mathrm{F}_{3}$, cuja correlação ao quadrado $\left(\mathrm{r}^{2}=0,25\right)$ foi inferior aos valores de VEM de cada um desses fatores $\left(\mathrm{VEM}_{\mathrm{F} 1}=0,32 ; \mathrm{VEM}_{\mathrm{F} 3}=0,45\right)$. Pode-se também verificar a validade discriminante dos fatores $F_{2}$ e $F_{3}$, que apresentaram valores de VEM $\left(\mathrm{VEM}_{\mathrm{F} 2}=0,41 ; \mathrm{VEM}_{\mathrm{F} 3}=0,45\right)$ superiores ao quadrado do coeficiente de correlação entre eles $\left(r^{2}=0,19\right)$. No entanto, ao se comparar a correlação ao quadrado $\left(r^{2}=0,67\right)$ dos fatores $F_{1}$ e $F_{2}$ com seus valores de VEM $\left(\mathrm{VEM}_{\mathrm{F} 1}=0,32 ; \mathrm{VEM}_{\mathrm{F} 2}=0,41\right)$, não foi observada a validade discriminante.

O IAT demonstrou ser capaz de distinguir os diferentes níveis de adição em Internet entre subgrupos populacionais estratificados por variáveis demográficas, socioeconômicas e de perfil de uso da Internet. Foi verificado que ser do sexo feminino, ser menor idade, pertencer a uma classe econômica

\section{Tabela 3}

Correlação dos itens com os fatores, alfa de Cronbach se o item for excluído do fator, alfa de Cronbach, variância extraída média (VEM) e confiabilidade composta (CC) dos fatores "Preocupação emocional e cognitiva com a Internet", "Problemas de gerenciamento de tempo" e "Problemas de desempenho".

\begin{tabular}{|c|c|c|c|c|c|}
\hline Item & $\begin{array}{l}\text { Correlação do item } \\
\text { com o fator }\end{array}$ & $\begin{array}{l}\text { Alfa de Cronbach se o item } \\
\text { for excluído do fator }\end{array}$ & $\begin{array}{l}\text { Alfa de } \\
\text { Cronbach }\end{array}$ & VEM & CC \\
\hline \multicolumn{6}{|c|}{ Preocupação emocional e cognitiva com a Internet } \\
\hline IAT 3 & 0,43 & 0,84 & 0,85 & 0,32 & 0,84 \\
\hline IAT 4 & 0,44 & 0,84 & & & \\
\hline IAT 9 & 0,45 & 0,84 & & & \\
\hline IAT 10 & 0,55 & 0,83 & & & \\
\hline IAT 11 & 0,65 & 0,82 & & & \\
\hline IAT 12 & 0,56 & 0,83 & & & \\
\hline IAT 13 & 0,53 & 0,84 & & & \\
\hline IAT 15 & 0,62 & 0,83 & & & \\
\hline IAT 18 & 0,52 & 0,84 & & & \\
\hline IAT 19 & 0,47 & 0,84 & & & \\
\hline IAT 20 & 0,62 & 0,83 & & & \\
\hline \multicolumn{6}{|c|}{ Problemas de gerenciamento de tempo } \\
\hline IAT 1 & 0,47 & 0,78 & 0,80 & 0,41 & 0,81 \\
\hline IAT 5 & 0,51 & 0,78 & & & \\
\hline IAT 7 & 0,58 & 0,76 & & & \\
\hline IAT 14 & 0,56 & 0,77 & & & \\
\hline IAT 16 & 0,63 & 0,75 & & & \\
\hline IAT 17 & 0,57 & 0,76 & & & \\
\hline \multicolumn{6}{|c|}{ Problemas de desempenho } \\
\hline IAT 2 & 0,45 & 0,76 & 0,73 & 0,45 & 0,71 \\
\hline IAT 6 & 0,61 & 0,57 & & & \\
\hline IAT 8 & 0,61 & 0,57 & & & \\
\hline Total & & & 0,906 & & \\
\hline
\end{tabular}

IAT: Internet Addiction Test. 
mais elevada, ser usuário frequente da Internet para acessar as redes sociais, jogar online, ver/ouvir filmes, músicas e vídeos e ter hábito noturno são condições associadas a maiores níveis de adição em Internet, conforme descrito na Tabela 4.

A escala IAT demonstrou validade de construto, uma vez que, dos quatro critérios analisados, três deles (fatorial, discriminante e teste de hipótese) apresentaram resultados dentro dos limites considerados satisfatórios.

\section{Validade de critério concorrente}

Foram observadas correlações significativas entre o escore do IAT e autopercepção sobre a adição em Internet $(r=0,644 ; \mathrm{p}<0,05)$, tempo de uso da Internet em dias úteis $(\mathrm{r}=0,427 ; \mathrm{p}<0,05)$ e em fins de semana $(r=0,424 ; \mathrm{p}<0,05)$, o que demonstrou evidência de validade de critério concorrente do instrumento.

\section{Confiabilidade (consistência interna e reprodutibilidade)}

$\mathrm{Na}$ Tabela 3, estão apresentadas as medidas da consistência interna da escala global do IAT, bem como dos fatores $F_{1}, F_{2}$ e $F_{3}$, cujos valores de alfa de Cronbach estão superiores a 0,70. Também estão apresentadas a correlação dos itens com o fator e o valor do alfa de Cronbach se o item for excluído.

\section{Tabela 4}

Teste de hipótese: comparação dos escores do Internet Addiction Test (IAT) segundo sexo, idade, classe econômica, uso de redes sociais, jogos online, ver/ ouvir filmes, músicas e vídeos e hábito noturno. Montes Claros, Minas Gerais, Brasil, 2016-2017.

\begin{tabular}{|c|c|c|c|c|c|}
\hline Variáveis & Média & DP & Mediana & IIQ & Valor de $p$ \\
\hline Sexo & & & & & 0,035 \\
\hline Masculino & 42,5 & 14,8 & 40,0 & 20,0 & \\
\hline Feminino & 43,8 & 15,3 & 41,0 & 22,0 & \\
\hline Idade (anos) & & & & & $<0,001$ \\
\hline$\leq 17$ & 46,5 & 15,4 & 45,0 & 22,0 & \\
\hline $18-24$ & 43,5 & 14,7 & 41,0 & 20,0 & \\
\hline$\geq 25$ & 37,0 & 13,7 & 34,0 & 18,0 & \\
\hline Classe econômica & & & & & $<0,001$ \\
\hline A & 46,8 & 14,4 & 45,0 & 19,0 & \\
\hline B & 44,0 & 15,2 & 41,5 & 20,0 & \\
\hline$C$ & 41,5 & 14,6 & 39,0 & 20,0 & \\
\hline $\mathrm{D} / \mathrm{E}$ & 42,5 & 15,6 & 39,0 & 24,0 & \\
\hline Uso frequente de redes sociais & & & & & $<0,001$ \\
\hline Não & 29,9 & 9,7 & 27,0 & 12,0 & \\
\hline Sim & 44,5 & 14,9 & 42,0 & 21,0 & \\
\hline Uso frequente de jogos online & & & & & $<0,001$ \\
\hline Não & 41,8 & 14,6 & 39,0 & 21,0 & \\
\hline $\operatorname{Sim}$ & 49,7 & 15,6 & 48,0 & 22,0 & \\
\hline Uso frequente para ver/ouvir filmes, músicas e vídeos & & & & & $<0,001$ \\
\hline Não & 36,6 & 13,7 & 33,0 & 18,0 & \\
\hline $\operatorname{Sim}$ & 45,7 & 14,8 & 43,0 & 20,0 & \\
\hline Uso frequente no período noturno & & & & & $<0,001$ \\
\hline Não & 35,9 & 13,8 & 32,0 & 19,0 & \\
\hline Sim & 44,6 & 14,9 & 42,0 & 21,0 & \\
\hline
\end{tabular}

DP: desvio padrão; IIQ: intervalo interquartílico. 
Em relação à reprodutibilidade, a amostra foi constituída de 320 estudantes, 157 dos quais eram do Ensino Médio e 163, do Ensino Superior. Os valores de CCI para os escores de cada fator e da escala geral do IAT foram iguais a $0,87\left(\mathrm{~F}_{1}\right), 0,84\left(\mathrm{~F}_{2}\right), 0,80\left(\mathrm{~F}_{3}\right)$ e 0,90 para o escore geral do IAT. O valor do coeficiente kappa foi igual a $0,73(\mathrm{p}<0,001)$ para a estimativa de concordância da categorização dos escores do IAT (mediano, problemático ou adicto).

\section{Discussão}

Este estudo avaliou as evidências de validade e confiabilidade do IAT em uma população de estudantes do ensinos Médio e Superior do norte de Minas Gerais. Confirmou-se a validade de construto, validade de critério e confiabilidade do IAT, corroborando a aplicabilidade dessa ferramenta em estudantes da região considerada. No cenário nacional, não foram identificados outros trabalhos que avaliaram conjuntamente as propriedades psicométricas desse instrumento. A realização de estudos que avaliem a validade desse construto tem sido sugerida desde a sua elaboração 15 .

A investigação da adição em Internet em estudantes é importante, uma vez que essa condição tem sido frequente no contexto estudantil e pode repercutir na vida cotidiana e na saúde dos jovens 12,18,22. O uso de instrumentos válidos e confiáveis que avaliem adequadamente o construto é necessário para levantar informações precisas e subsidiar estratégias de intervenção.

O conceito de adição em Internet é frequentemente utilizado pelas comunidades leiga e científica com uma diversidade de abordagens. Essa condição tem sido caracterizada por padrões distintos de comportamento, que podem abordar o uso excessivo, uso problemático, controle inadequado e vício da Internet, que não refletem necessariamente o mesmo problema. Adicionalmente, pode incluir outros comportamentos problemáticos no uso da Internet, como jogos, redes sociais e pornografia 17,34.

Este estudo identificou um modelo de medida para o IAT com estrutura trifatorial, cujos fatores são denominados "preocupação emocional e cognitiva com a Internet", "problemas de gerenciamento de tempo" e "problemas de desempenho". Esse modelo atendeu às expectativas de qualidade de ajuste referenciado por Marôco 30, indicando que representa adequadamente as correlações entre os itens e seus respectivos fatores. O modelo ajustado confirma a possibilidade do uso de diferentes modelos dimensionais do IAT, para além do uso da versão original.

A identificação das três dimensões do construto estudado possibilita rastrear a adicção em Internet em suas distintas manifestações, a fim de estabelecer medidas específicas de prevenção, controle e tratamento. Tal fato é pertinente diante das ponderações que devem ser consideradas ao utilizar a designação de dependência atribuída ao uso da Internet, que muitas vezes é apropriada para uma parcela minoritária de indivíduos. Há ainda a limitação em distinguir se a adição em Internet corresponde a uma condição distinta ou a uma manifestação de um transtorno psíquico, com o reconhecimento da existência de consequências negativas relacionadas ao uso inapropriado desta ferramenta 34 . Há que se considerarem as conotações estigmatizantes da sociedade relacionadas à condição de dependência 35, o que pode prejudicar na sensibilização e na conscientização sobre o problema.

Outros trabalhos evidenciaram a estrutura trifatorial do IAT em estudantes 13, todavia o agrupamento dos itens que compõem cada um dos fatores diferiu. Observou-se que apenas o fator "problemas de gerenciamento de tempo" é comum à maioria. Estudo realizado em países europeus 23 identificou os fatores "problemas de gerenciamento de tempo", "conflitos emocionais" e "modificação do humor” como manifestação da adição em Internet. Estudos como o de Sung et al. 19 indicaram, além dos "problemas de gerenciamento de tempo", a presença de "comportamentos de abstinência" e de "perda de controle". A análise desenvolvida em Hong Kong 36 identificou as dimensões "isolamento e problemas sociais", "problemas de gerenciamento de tempo" e a "substituição da realidade".

Trabalhos diversos identificaram diferentes estruturas dimensionais para o IAT. Faraci et al. 37 encontraram uma estrutura fatorial de duas dimensões: "preocupação emocional e cognitiva com a Internet" e "perda de controle e interferência com a vida diária”. Dois autores identificaram uma estrutura com seis dimensões, embora diferentes entre si. Ferraro et al. 38, na Itália, com as dimensões "controle de tempo comprometido", "qualidade de vida social comprometida", "qualidade de vida individual comprometida", "uso excitatório", "uso compensatório” e "carreira acadêmica/trabalhista 
comprometida"; Widyanto \& McMurran 39, no Reino Unido, com as dimensões "falta de controle", "uso excessivo", "negligenciar a vida social", "saliência", "antecipação" e "negligenciar trabalho". Tal fato pode se dever às diferenças culturais existentes entre os países relacionadas à própria Internet e ao seu uso, além de questões metodológicas 17.

No contexto da AFC, a validade convergente ocorre quando os itens que são reflexos de um fator o saturam fortemente; por isso, os itens indicadores de um construto específico devem possuir uma elevada proporção de variância comum 31. O modelo de medida ajustado obteve valores de VEM abaixo dos níveis aceitáveis, o que pode ter sido influenciado pela heterogeneidade da amostra: áreas do conhecimento, sistemas de ensino (público e privado) e níveis educacionais (Médio e Superior) diferentes. Esses resultados apontam para uma baixa proporção da variância dos itens, explicada pelo construto ao qual pertencem. Apesar de os valores de CC apresentarem-se satisfatórios, não houve, no presente estudo, evidência robusta quanto à validade convergente do IAT. Estudo prévio $17 \mathrm{fez}$ ponderações em relação à elaboração de alguns itens do IAT, que não parece refletir um processo psicométrico rigoroso, pois viola a regra geral de que todo item deve refletir conteúdo específico e um único comportamento mental específico. Isso pode explicar os baixos valores de VEM encontrados. É importante destacar também que a heterogeneidade da amostra, composta por estudantes de diferentes áreas do conhecimento, sistemas de ensino (público e privado) e níveis educacionais (Médio e Superior), pode ter influenciado neste resultado.

A validade discriminante do IAT foi verificada, já que a variância compartilhada entre os constructos não foi maior que suas respectivas variâncias extraídas. Os resultados obtidos sugerem que os itens de um fator não estão mais fortemente correlacionados com os de outro fator, sugerindo que os fatores são distintos entre si.

Observaram-se diferenças significativas na comparação dos escores do IAT entre os subgrupos de estudantes, confirmando a hipótese de que a escala IAT é capaz de discriminar subgrupos que teoricamente apresentariam níveis diferentes de adição em Internet. Esses resultados corroboram os achados de Chang \& Law 36 e Sung et al. 19, ambos em amostras asiáticas, apesar de apresentarem diferenças metodológicas. Já em estudo realizado com indivíduos diagnosticados clinicamente com dependência da Internet, os escores do IAT não foram correlacionados significativamente com a gravidade clínica e a duração da doença 24 . Este aspecto demonstra a necessidade de uma avaliação clínica para o diagnóstico da adição em Internet, visto que o IAT é uma ferramenta de rastreio.

Para a validade de critério concorrente, recomenda-se a comparação entre os escores do instrumento sob validação e de uma escala "padrão-ouro". Entretanto, esta análise foi realizada por meio da comparação dos escores do IAT com outras referências externas, que teoricamente deveriam correlacionar-se ao construto ("autopercepção da adição em Internet" e "tempo de utilização de Internet"). Há que se ponderar que os indivíduos adictos tendem a ter maior tempo de conexão, mas nem todos que utilizam a Internet por muito tempo são adictos. Estudos prévios também atestaram a validade concorrente do IAT comparando com escala que avalia o mesmo construto 22 e com escalas que avaliam construtos diferentes, como solidão 13 e sintomas depressivos 17 .

No que se refere à confiabilidade do IAT, observaram-se índices satisfatórios de consistência interna para a escala global e para os três fatores, o que evidencia a homogeneidade dos itens e a sua capacidade de medir sem erros 40 . A confiabilidade do IAT também foi observada em estudos realizados com estudantes de diferentes nacionalidades 12,14,17. Com relação à reprodutibilidade do instrumento, foram observados índices de concordância adequados, o que é compatível com o verificado em suas versões da Espanha 18 e da Malásia 22. Esses achados pressupõem que as respostas dadas ao instrumento em diferentes momentos são confiáveis, o que indicou estabilidade temporal do IAT 30.

O presente estudo tem como limitação o uso do método de máxima verossimilhança para o ajuste do modelo de medida, o qual requer normalidade univariada e multivariada, além de variáveis em escala contínua 27 . No entanto, os itens do IAT estão numa escala de mensuração ordinal com cinco categorias, ou seja, são variáveis que não comportam uma análise paramétrica. Vale ressaltar que os coeficientes de assimetria e curtose apresentaram valores que indicam uma aproximação de distribuição normal e que o método de máxima verossimilhança é robusto quando esses parâmetros não são muito elevados, ainda que as variáveis estejam em uma escala ordinal 31. Outra limitação do estudo refere-se à validade de critério concorrente do IAT, sendo mais adequado correlacionar com outro instrumento validado que mensure o mesmo construto. 
O instrumento IAT apresentou propriedades psicométricas satisfatórias de sensibilidade psicométrica, validade de construto, validade de critério concorrente e confiabilidade. A versão do IAT, traduzida e adaptada para o idioma português, a partir desse estudo, pode ser considerada válida (com ressalvas para a validade convergente) e confiável para avaliar a adicção em Internet em pesquisas epidemiológicas entre estudantes do Ensino Médio e Superior brasileiros.

\section{Colaboradores}

A. B. Brito, L. Pinho, M. F. S. F. Brito, R. B. Messias e M. F. Silveira contibuíram com o planejamento do estudo, coleta de dados, escrita e revisão final do artigo. K. D. P. Brito, C. A. O. Rodrigues e V. M. C. P. Reis contribuíram com a coleta dos dados e escrita do artigo.

\section{Informações adicionais}

ORCID: Alexandre Botelho Brito (0000-00032628-2117); Lucinéia de Pinho (0000-0002-29475806); Maria Fernanda Santos Figueiredo Brito (0000-0002-6133-9855); Romerson Brito Messias (0000-0002-4781-5050); Kewla Dias Pires Brito (0000-0003-1149-6753); Carolina Amaral Oliveira Rodrigues (0000-0003-1804-619X); Vivianne Margareth Chaves Pereira Reis (0000-0001-8797-2678); Marise Fagundes Silveira (0000-0002-8821-3160).

\section{Agradecimentos}

Ao apoio e financiamento via: Programa de Pósgraduação em Ciências da Saúde da Universidade Estadual de Montes Claros, Coordenação de Aperfeiçoamento de Pessoal de Nível Superior (CAPES; bolsa de Doutorado de A. B. Brito), Fundação de Amparo à Pesquisa do Estado de Minas Gerais (FAPEMIG; financiamento), Instituto Federal do Norte de Minas Gerais (IFNMG; financiamento) e Conselho Nacional de Desenvolvimento Científico e Tecnológico (CNPq; bolsa de iniciação científica).

\section{Referências}

1. Lee Y-H, Ko C-H, Chou C. Re-visiting Internet addiction among Taiwanese students: a crosssectional comparison of students' expectations, online gaming, and online social interaction. J Abnorm Child Psychol 2015; 43:589-99.

2. Younes F, Halawi G, Jabbour H, El Osta N, Karam L, Hajj A, et al. Internet addiction and relationships with insomnia, anxiety, depression, stress and self-esteem in university students: cross-sectional designed study. PLoS One 2016; 11:e0161126.

3. Hinić D. Problems with 'Internet addiction' diagnosis and classification. Psychiatr Danub 2011; 23:145-51.

4. Lee K, Lee H-K, Gyeong H, Yu B, Song Y-M, Kim D. Reliability and validity of the Korean version of Internet Addiction Test among college students. J Korean Med Sci 2013; 28:763-8.

5. Seyrek S, Cop E, Sinir H, Ugurlu M, Şenel S. Factors associated with Internet addiction: cross-sectional study of Turkish adolescents. Pediatr Int 2017; 59:218-22.

6. Ostovar S, Allahyar N, Aminpoor H, Moafian F, Nor MBM, Griffiths MD. Internet addiction and psychosocial risks (depression, anxiety and loneliness) among Iranian adolescents and young adults: a structural equation model in a cross-sectional study. Int J Ment Health Addict 2016; 14:257-67.

7. Lin P-C, Kuo S-Y, Lee P-H, Sheen T-C, Chen S-R. Effects of Internet addiction on heart rate variability in school-aged children. J Cardiovasc Nurs 2014; 29:493-8.

8. Khoury JM, de Freitas AAC, Roque MAV, Albuquerque MR, das Neves MCL, Garcia FD. Assessment of accuracy of a new tool for the screening of smartphone addiction. PLoS One 2017; 12:e0176924. 
9. Li W, Garland EL, Howard MO. Family factors in Internet addiction among Chinese youth: a review English-and Chinese-language studies. Comput Human Behav 2014; 31:393-411.

10. Batista JRM. Adição à Internet: análise de seu significado e suas relações com a qualidade de vida [Dissertação de Mestrado]. João Pessoa: Universidade Federal da Paraíba; 2011.

11. Pontes HM, Griffiths MD. Internet addiction disorder and internet gaming disorder are not the same. J Addict Res Ther 2014; 5:e124.

12. Jelenchick LA, Becker T, Moreno MA. Assessing the psychometric properties of the Internet Addiction Test (IAT) in US college students. Psychiatry Res 2012; 196:296-301.

13. Neelapaijit A, Pinyopornpanish M, Simcharoen S, Kuntawong P, Wongpakaran N, Wongpakaran T. Psychometric properties of Thai version internet addiction test. BMC Res Notes 2018; 11:69.

14. Wu X-S, Zhang Z-H, Zhao F, Wang W-J, Li Y-F, Bi L, et al. Prevalence of Internet addiction and association with social support and other related factors among adolescents in China. J Adolesc 2016; 52:103-11.

15. Young KS. Internet addiction: the emergence of new clinical disorder. Cyberpsychol Behav 1998; 1:237-44.

16. Conti MA, Jardim AP, Hearst N, Cordás TA, Tavares H, Abreu CN. Avaliação da equivalência semântica e consistência interna de uma versão em português do Internet Addiction Test (IAT). Arch Clin Psychiatry 2012; 39:10610.

17. Pontes HM, Patrao IM, Griffiths MD. Portuguese validation the Internet Addiction Test: empirical study. J Behav Addict 2014; 3:10714.

18. Fernández-Villa T, Molina AJ, García-Martín M, Llorca J, Delgado-Rodríguez M, Martín V. Validation and psychometric analysis of Internet Addiction Test in Spanish among college students. BMC Public Health 2015; 15:953.

19. Sung M, Shin Y-M, Cho S-M. Factor structure of Internet Addiction Scale and its associations with psychiatric symptoms for Korean adolescents. Community Ment Health J 2014; 50:612-8.

20. Lu X, Yeo KJ. Psychometric properties of Internet Addiction Test in a sample of Malaysian undergraduate students. Psicol Educ (Madrid) 2015; 21:17-25.

21. Khazaal Y, Billieux J, Thorens G, Khan R, Louati Y, Scarlatti E, et al. French validation of internet addiction test. Cyberpsychol Behav 2008; 11:703-6.

22. Chong Guan N, Isa SM, Hashim AH, Pillai SK, Harbajan Singh MK. Validity of Malay version of Internet Addiction Test: a study on a group of medical students in Malaysia. Asia Pac J Public Health 2015; 27:NP2210-9.

23. Widyanto L, Griffiths MD, Brunsden V. A psychometric comparison of Internet Addiction Test, the Internet-Related Problem Scale, and self-diagnosis. Cyberpsychol Behav Soc Netw 2011; 14:141-9.
24. Kim SJ, Park D-H, Ryu S-H, Yu J, Ha JH. Usefulness of Young's Internet addiction test for clinical populations. Nord J Psychiatry 2013; 67:393-9.

25. Farias Júnior JC, Loch MR, Lima Neto AJ, Sales JM, Ferreira FELL. Reprodutibilidade, consistência interna e validade de construto do KIDSCREEN-27 em adolescentes brasileiros. Cad Saúde Pública 2017; 33:e00131116.

26. Yayan EH, Arikan D, Saban F, Gürarslan Baş N, Özel Özcan Ö. Examination of correlation between Internet addiction and social phobia in adolescents. West J Nurs Res 2017; 39:124054.

27. Kline RB. Principles and practice of structural equation modeling. New York: Guilford Publications; 2015.

28. Área Técnica de Saúde Bucal, Departamento de Atenção Básica, Secretaria de Políticas de Saúde, Ministério da Saúde. Projeto SB2000: condições de saúde bucal da população brasileira no ano 2000. Manual do examinador. Brasília: Ministério da Saúde; 2001.

29. Dziuban CD, Shirkey EC. When is correlation matrix appropriate for factor analysis? Some decision rules. Psychol Bull 1974; 81:358-61.

30. Marôco JP, Campos JADB, Vinagre MG, Pais-Ribeiro JL. Adaptação transcultural Brasil-Portugal da Escala de Satisfação com o Suporte Social para estudantes do ensino superior. Psicol Reflex Crít 2014; 27:247-56.

31. Marôco J. Análise de equações estruturais: fundamentos teóricos, software e aplicação. Pêro Pinheiro: ReportNumber; 2010.

32. Hair JF, Black WC, Babin BJ, Anderson RE, Tatham RL. Análise multivariada de dados. Porto Alegre: Bookman Editora; 2009.

33. Shrout PE, Fleiss JL. Intraclass correlations: uses assessing rater reliability. Psychol Bull 1979; 86:420-8.

34. Starcevic V, Aboujaoude E. Internet addiction: reappraisal of increasingly inadequate concept. CNS Spectr 2017; 22:7-13.

35. Starčević V. Internet gaming disorder: inadequate diagnostic criteria wrapped in constraining conceptual model. J Behav Addict 2017; 6:110-3.

36. Chang MK, Law SPM. Factor structure for Young's Internet Addiction Test: a confirmatory study. Comput Human Behav 2008; 24:2597-619.

37. Faraci P, Craparo G, Messina R, Severino S. Internet Addiction Test: which is the best factorial solution? J Med Internet Res 2013; 15:e225.

38. Ferraro G, Caci B, D’Amico A, Di Blasi M. Internet Addiction Disorder: un contributo di ricerca. Psicotech 2006; (1):7-25.

39. Widyanto L, McMurran M. Psychometric properties of the internet addiction test. Cyberpsychol Behav 2004; 7:443-50.

40. Pasquali L. Psicometria: teoria dos testes na psicologia e educação. Petrópolis: Editora Vozes; 2017. 
Abstract

The study aimed to assess the psychometric properties of the Brazilian Portuguese version of the Internet Addiction Test (IAT) in students. The methodological study population consisted of secondary and university students from Montes Claros, Minas Gerais State, Brazil. The following parameters were assessed: psychometric sensitivity, construct validity (factor, convergent, discriminant, and test of hypothesis), criterion validity (concurrent), and reliability (internal consistency and reproducibility). A total of 2,519 students participated, 29.6\% of whom were secondary school enrollees. A three-factor measurement model was adjusted ("emotional and cognitive preoccupation with the Internet", "problems in managing time", and "performance problems"), which obtained satisfactory adequacy indices and stable structure in the independent subsamples. Convergent validity was close to the recommended level (mean extracted variation $=0.32,0.41$, and 0.45 and compound reliability values $=0.84,0.81$, and 0.71 ), and discriminant and concurrent validities were adequate. Internal consistency was adequate (alpha $=0.906$ ), as was reproducibility (kappa $=0.73$ and intraclass correlation coefficient $=0.90$ ). The Portuguese-language version of the IAT presented satisfactory levels of validity, reliability, and stability in independent samples of students.

Validation Study; Internet; Students

\section{Resumen}

El objetivo fue evaluar las propiedades psicométricas de la versión traducida al portugués del Internet Addiction Test (IAT) en estudiantes. Estudio metodológico, cuya población estuvo constituida por estudiantes de enseñanza media y superior de Montes Claros, Minas Gerais, Brasil. Se evaluaron: sensibilidad psicométrica, validez del constructo (factorial, convergente, discriminante y test de hipótesis), validez de criterio (concurrente) y confiabilidad (consistencia interna y reproducibilidad). Participaron 2.519 estudiantes, siendo un $29,6 \%$ de enseñanza media. Se ajustó un modelo de medida trifactorial: "preocupación emocional y cognitiva con Internet", "problemas de gestión de tiempo" y "problemas de desempeño", con el que se obtuvieron indices satisfactorios de adecuación $y$ estructura estable en las submuestras independientes. La validez convergente estuvo cercana a lo recomendado (variancia extraída media $=0,32$, 0,41 y 0,45y valores de confiabilidad compuest $a=$ $0,84,0,81$ y 0,71), la validez, tanto discriminante como concurrente, fueron adecuadas. La consistencia interna fue adecuada (alfa =0,906), así como la reproducibilidad ( $k a p p a=0,73$ y coeficiente de correlación intraclase $=0,90$ ). La versión traducida al portugués del IAT presentó niveles satisfactorios de validez, confiabilidad y estabilidad en muestras independientes de estudiantes.

Estudio de Validación; Internet; Estudiantes
Recebido em 31/Out/2019

Versão final reapresentada em 26/Dez/2020

Aprovado em 19/Fev/2021 